\title{
ON UNCONDITIONAL CONVERGENCE IN NORMED VECTOR SPACES
}

\author{
T. H. HILDEBRANDT
}

Suppose $X$ is a complete normed vector or Banach space of elements $x$. Orlicz ${ }^{1}$ has given the following two definitions of unconditional convergence of an infinite series $\sum_{n} x_{n}$ of elements from $X$ and proved their equivalence:

A. $\sum_{n} x_{n}$ is unconditionally convergent if and only if any rearrangement of the series is convergent.

B. $\sum x_{n}$ is unconditionally convergent if and only if $\sum_{k} x_{n_{k}}$ converges, where $\left\{x_{n_{k}}\right\}$ is any subsequence of $\left\{x_{n}\right\}$.

Pettis ${ }^{2}$ has shown that either of these conditions is equivalent to the statement:

C. Every subseries of $\sum_{n} x_{n}$ is weakly convergent to an element of $X$, that is, $\left\{x_{n_{k}}\right\}$ implies the existence of an element $x_{\sigma}$ such that, for every $\bar{x}$ of the conjugate space $\bar{X}, \sum_{k} \bar{x}\left(x_{n_{k}}\right)=\bar{x}\left(x_{\sigma}\right)$.

In proving this equivalence, he shows that condition $\mathrm{C}$ implies the following:

D. $\lim _{n} \sum_{m=n}^{\infty} \bar{x}\left(x_{m}\right)=0$ uniformly for $\|\bar{x}\|=1$.

E. H. Moore ${ }^{3}$ has shown that for real, complex or quaternionic numbers, absolute and therefore unconditional convergence is equivalent to the following definition of convergence:

Let $\sigma$ be any finite subset $n_{1}, \cdots, n_{k}$ of the positive integers, and denote $\sum_{i=1}^{k} x_{n_{i}}$ by $\sum_{\sigma} x_{n}$. Then

E. $\sum x_{n}$ converges in the $\sigma$-sense, if $\lim _{\sigma} \sum_{\sigma} x_{n}$ exists, where the limit is the Moore-Smith limit, and $\sigma_{1} \geqq \sigma_{2}$ means that $\sigma_{1}$ contains all of the numbers in $\sigma_{2}{ }^{4}$

Obviously the Moore-Smith limit can be extended to normed vector spaces, and the fundamental properties carry over. It is the purpose of this note to show that convergence in the $\sigma$-sense is equivalent to each of the conditions $\mathrm{A}, \mathrm{B}$, and $\mathrm{D}$, that is, $\mathrm{A}, \mathrm{B}, \mathrm{D}$ and $\mathrm{E}$ are equivalent definitions of unconditional convergence, to which the

1 Ueber unbedingte Konvergenz in Funktionenräumen, Studia Mathematica, vol. 4 (1933), pp. 33-38. 282.

2 Integration in vector spaces, Transactions of this Society, vol. 44 (1938), pp. 281-

${ }^{3}$ General Analysis, Memoirs of the American Philosophical Society, vol. 1, part 2, 1939, p. 63.

${ }^{4}$ See Alaoglu, Annals of Mathematics, (2), vol. 41 (1940), p. 259, where a similar definition for weak unconditional convergence is given. 
Pettis result adds $\mathrm{C}$ as a fifth equivalent definition. We consider definition $\mathrm{E}$ the most elegant of the definitions of unconditional convergence. ${ }^{5}$

A is equivalent to E. Assume that every rearrangement of $\sum x_{n}$ is convergent and suppose if possible $\lim _{\sigma} \sum_{\sigma} x_{n}$ is not equal to $x=\sum_{n} x_{n}$, summed in its natural order. Then there exists an $e>0$, such that for every $\sigma$ there exists a $\sigma^{\prime} \geqq \sigma$ such that $\left\|x-\sum_{\sigma^{\prime}} x_{n}\right\| \geqq e$. Let $n_{0}$ be chosen so that for $n \geqq n_{0}$ we have $\left\|x-\sum_{1}^{n} x_{m}\right\|<e / 2$. Let $\sigma_{1}$ be the set $1,2, \cdots, n_{0}$, and $\sigma_{1}^{\prime}$ chosen so that $\left\|x-\sum_{\sigma_{1}^{\prime}} x_{n}\right\| \geqq e$. Let $\sigma_{2}$ include all of the integers less than or equal to any integer in $\sigma_{1}^{\prime}$. Repeating the process produces a $\sigma_{2}^{\prime}$, a $\sigma_{3}$, and so on, and defines a rearrangement of $\sum x_{n}$, namely, $\sigma_{1}, \sigma_{1}^{\prime}-\sigma_{1}, \sigma_{2}-\sigma_{1}^{\prime}, \ldots$ (where $\sigma-\sigma^{\prime}$ means the elements of $\sigma$ not in $\sigma^{\prime}$ ), which is not a convergent series since

$$
\left\|\sum_{\sigma_{m}} x_{n}-\sum_{\sigma_{m^{\prime}}} x_{n}\right\|=\left\|\sum_{\sigma_{m^{\prime}}-\sigma_{m}} x_{n}\right\| \geqq e / 2 \text {. }
$$

Since $\lim _{\sigma} \sum_{\sigma} x_{n}$ is unique when it exists, it follows at once that every rearrangement of $\sum_{n} x_{n}$ converges to the same limit. ${ }^{6}$

Conversely, suppose $\sum_{\sigma} x_{n}$ approaches a limit $x$, that is, for every $e>0$, there exists a $\sigma_{e}$ such that if $\sigma \geqq \sigma_{e}$ then $\left\|\sum_{\sigma} x_{n}-x\right\| \leqq e$. Let $\left\{x_{n_{k}}\right\}$ be any rearrangement of $\left\{x_{n}\right\}$. Then we need only to choose $k_{e}$ so that the set of integers $n_{1}, \cdots, n_{k_{e}}$ includes all of the integers in $\sigma_{e}$ to be assured that for $k^{\prime} \geqq k_{e}$ it is true that

$$
\left\|\sum_{k=1}^{k^{\prime}} x_{n_{k}}-x\right\| \leqq e .
$$

$\mathrm{B}$ is equivalent to $\mathrm{E}$. Suppose every subseries $\sum_{k} x_{n_{k}}$ converges but $\lim _{\sigma} \sum_{\sigma} x_{n}$ does not exist, that is, there exists an $e>0$ such that for every $\sigma$ there exist $\sigma^{\prime}, \sigma^{\prime \prime} \geqq \sigma$ such that $\left\|\sum_{\sigma^{\prime}} x_{n}-\sum_{\sigma^{\prime}} x_{n}\right\|>e$. If $\sigma^{\prime}+\sigma^{\prime \prime}$ denotes the set including all elements of $\sigma^{\prime}$ and $\sigma^{\prime \prime}$, then either $\left\|\sum_{\sigma^{\prime}} x_{n}-\sum_{\sigma^{\prime}+\sigma^{\prime}} x_{n}\right\| \geqq e / 2$ or $\left\|\sum_{\sigma^{\prime}} x_{n}-\sum_{\sigma^{\prime}+\sigma^{\prime}} x_{n}\right\| \geqq e / 2$, that is, we can assume $\sigma^{\prime} \geqq \sigma^{\prime \prime} \geqq \sigma$. We obtain a nonconvergent subseries as follows: Take $\sigma_{1}=1$. This gives rise to $\sigma_{1}^{\prime} \geqq \sigma_{1}^{\prime \prime} \geqq \sigma_{1}$ so that $\left\|\sum_{\sigma_{1}^{\prime}} x_{n}-\sum_{\sigma_{1}^{\prime \prime}} x_{n}\right\|$ $>e$. Let $n_{1}, \cdots, n_{k_{1}}$ be the elements of $\sigma_{1}^{\prime}-\sigma_{1}^{\prime \prime}$. Take $\sigma_{2}=\sigma_{1}^{\prime}$, and $n_{k_{1}+1}, \cdots, n_{k_{2}}$ to be the elements of $\sigma_{2}^{\prime}-\sigma_{2}^{\prime \prime}$. Proceeding in this manner we get a series $x_{n_{1}}+x_{n_{2}}+\cdots+x_{n_{k_{1}}}+\cdots+x_{n_{k 2}}+\cdots$ such that $\left\|\sum_{k_{m+1}}^{k_{m+1} x_{n_{k}}}\right\|>e$, that is, one which is not convergent.

${ }^{5}$ In view of the results of Orlicz and Pettis, it would be sufficient to prove that D implies $\mathrm{E}$ implies $\mathrm{A}$. For the sake of completeness and elegance we have preferred to prove each equivalence separately.

${ }^{6}$ See Orlicz, Studia Mathematica, vol. 1 (1929), p. 242. 
Conversely if $\lim _{\sigma} \sum_{\sigma} x_{n}$ exists and $\left\{x_{n_{k}}\right\}$ is any subsequence of $x_{n}$, then if $k_{e}$ is chosen so that $n_{k_{e}}$ is larger than any integer appearing in the $\sigma_{e}$ involved in the definition of the limit, it will be certain that for $k^{\prime} \geqq k_{e}$ and any $l$

$$
\left\|\sum_{k=k^{\prime}}^{k^{\prime}+l} x_{n_{k}}\right\| \leqq 2 e,
$$

that is, $\sum_{k} x_{n_{k}}$ is convergent.

$\mathrm{D}$ is equivalent to $\mathrm{E}$. Suppose $\lim _{n} \sum_{m=n}^{\infty}\left|\bar{x}\left(x_{m}\right)\right|=0$ uniformly for $\|\bar{x}\|=1$. We demonstrate that $\lim _{\sigma_{1}, \sigma_{2}}|| \sum_{\sigma_{1}} x_{n}-\sum_{\sigma_{2}} x_{n} \|=0$. This is obviously equivalent to showing that $\lim _{\sigma_{1} \geqq \sigma_{2}}\left\|\sum_{\sigma_{1}} x_{n}-\sum_{\sigma_{2}} x_{n}\right\|=0$, since

$$
\left\|\sum_{\sigma_{1}} x_{n}-\sum_{\sigma_{2}} x_{n}\right\| \leqq\left\|\sum_{\sigma_{1}} x_{n}-\sum_{\sigma_{1}+\sigma_{2}} x_{n}\right\|+\left\|\sum_{\sigma_{2}} x_{n}-\sum_{\sigma_{1}+\sigma_{2}} x_{n}\right\| .
$$

Let $n_{e}$ be such that for $n \geqq n_{e}$ we have $\sum_{n}^{\infty}\left|\bar{x}\left(x_{m}\right)\right| \leqq e$ if $\|\bar{x}\|=1$, and take $\sigma_{e}=1,2, \cdots, n_{e}$ with $\sigma_{1} \geqq \sigma_{2} \geqq \sigma_{e}$. Then $\sum_{\sigma_{1}-\sigma_{2}}\left|\bar{x}\left(x_{n}\right)\right| \leqq e$ and so $\left|\bar{x}\left(\sum_{\sigma_{1}-\sigma_{2}} x_{n}\right)\right| \leqq e$. Since this is uniform for $\|\bar{x}\|=1$, it follows that

$$
\left\|\sum_{\sigma_{1}-\sigma_{2}} x_{n}\right\|=1 . u . b .\left[\left|\bar{x}\left(\sum_{\sigma_{1}-\sigma_{2}} x_{n}\right)\right| \text { for }\|\bar{x}\|=1\right] \leqq e .
$$

Conversely suppose $\lim _{\sigma} \sum_{\sigma} x_{n}$ exists. Then for $\sigma_{1} \geqq \sigma_{2} \geqq \sigma_{e}$ we have $\left\|\sum_{\sigma_{1}-\sigma_{2}} x_{n}\right\| \leqq e$. Take $n_{e}$ greater than the largest integer in $\sigma_{e}$. Let $m \geqq n_{e}$ and $\|\bar{x}\|=1$. Denote by $\sigma_{1 \bar{x}}$ those of the integers $m, m+1, \cdots$, $m+k$, for which $\bar{x}\left(x_{n}\right) \geqq 0$, and by $\sigma_{2 \bar{x}}$ the integers for which $\bar{x}\left(x_{n}\right)<0$. Then

$$
\begin{aligned}
& \sum_{m}^{m+k}\left|\bar{x}\left(x_{n}\right)\right|=\sum_{\sigma_{1} \bar{x}}\left|\bar{x}\left(x_{n}\right)\right|+\sum_{\sigma_{2} \bar{x}}\left|\bar{x}\left(x_{n}\right)\right| \\
& =\left|\bar{x}\left(\sum_{\sigma_{1} \bar{x}}\left(x_{n}\right)\right)\right|+\left|\bar{x}\left(\sum_{\sigma_{2} \bar{x}} x_{n}\right)\right| \leqq\left\|\sum_{\sigma_{1} \bar{x}} x_{n}\right\|+\left\|\sum_{\sigma_{2} \bar{x}} x_{n}\right\| \leqq 2 e ;
\end{aligned}
$$

that is, $\lim _{n} \sum_{n}^{\infty}\left|\bar{x}\left(x_{n}\right)\right|=0$, uniformly for $\|\bar{x}\|=1$. A similar procedure would take care of the case in which $\bar{x}(x)$ were complex valued.

We note that condition $\mathrm{D}$ is equivalent to the statement that the linear operations (functionals) $\bar{x}$ for which $\|\bar{x}\|=1$ map the sequence $x_{n}$ on a compact subset of $l$, the space of absolutely convergent series, that is, any unconditionally convergent series can be interpreted as a completely continuous transformation on the adjoint space $\bar{X}$ to the space $l .^{7}$

${ }^{7}$ See Dunford, Transactions of this Society, vol. 44 (1938), p. 322. 
Further it can be deduced from condition B that if $x(\sigma)=\sum_{k} x_{n_{k}}$ where $\sigma=n_{1}, n_{2}, \cdots$ then $x(\sigma)$ is a completely additive set function on subsets of the integers to the space $X$.

E. H. Moore, in the reference mentioned above, considers the case in which the set of integers $1,2, \cdots, n, \cdots$ is replaced by a general set: $\mathfrak{B}$, and then defines a general sum $\sum x(p)$ by an obvious generalization. He shows that if $\sum x(p)$ exists, then $x(p)$ is zero except at a denumerable set of elements $p_{1}, \cdots, p_{n}, \cdots$ and $\sum_{n}\left|x\left(p_{n}\right)\right|$ exists. This result is extensible to the case where $x$ is on $\mathfrak{P}$ to a linear normed complete space in the form:

If $x$ is on $\mathfrak{B}$ to $X$ and if $\sum x(p)$ exists in the sense that $\lim _{\sigma} \sum_{\sigma} x(p)$ exists, where the $\sigma$ are finite subsets of $\mathfrak{B}$, then $x(p)$ differs from zero at most at a denumerable set of elements $p_{1}, \cdots, p_{n}, \cdots$ and $\sum x\left(p_{n}\right)$ is unconditionally convergent.

The first part of this theorem depends on the fact, easily derived by a slight change in the proof of "E implies D" above, that if $\sum_{p} x(p)$ exists then $\lim _{\sigma} \sum_{\sigma}\left|\bar{x}\left(x_{p}\right)\right|$ exists uniformly for $\|\bar{x}\|=1$, that is, for every $e>0$ there exists a $\sigma_{e}$ such that if $\sigma_{1} \geqq \sigma_{2} \geqq \sigma_{e}$ and $\|\bar{x}\|=1$ then $\sum_{\sigma_{1}-\sigma_{2}}\left|\bar{x}\left(x_{p}\right)\right| \leqq e$. Let $\mathfrak{B}_{0}$ be the sum of the sets $\sigma_{e}$ for $e=1 / n$. This set will be denumerable. If $p$ is not of $\mathfrak{P}_{0}$ then $\left|\bar{x}\left(x_{p}\right)\right| \leqq 1 / n$ for all $n$ and $\|\bar{x}\|=1$, that is, $x_{p}=0$. The second part of the theorem is obvious.

UNIVERSITY OF MichigaN 